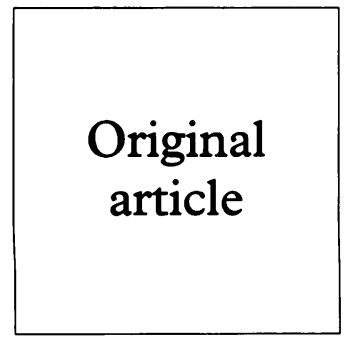

\section{Prior fluconazole exposure as an independent risk factor for fluconazole resistant candidosis in HIV positive patients: a case-control study}

\author{
Jonathan D Cartledge, Jennifer Midgley, Brian G Gazzard
}

\begin{abstract}
Objective: To determine if prior fluconazole exposure was an independent risk factor for fluconazole resistant candidosis in HIV positive patients.

Methods: Twenty five HIV positive cases with fluconazole resistant oral candidosis were matched by CD4 lymphocyte count and time since first episode of candidosis to $25 \mathrm{HIV}$ positive controls with susceptible candidosis. For each individual a history of prior azole prescription was compiled from computerised pharmacy records and review of case notes.

Results: The total days of prior azole therapy prescribed was significantly greater for cases than controls. These differences were attributable to prescriptions for secondary prophylaxis against recurrent candidosis, the cases having received significantly longer continuous azole prophylaxis than controls, with no difference in days of prior azole therapy remaining between the two groups if prophylactic prescriptions were excluded. The total cumulative dose of fluconazole received was significantly higher for cases than controls, though mean daily fluconazole doses did not differ significantly between the two groups.

Conclusion: Even after controlling for degree of immunosuppression and duration of recurrent candidosis, the association between prior azole exposure and fluconazole resistant candidosis remains significant and largely reflects differences in the prescription of secondary antifungal prophylaxis.
\end{abstract}

(Genitourin Med 1997;73:471-474)

Keywords: fluconazole; candidosis; HIV; risk factor

\section{Introduction}

A number of authors have reported cases of fluconazole resistant candidosis in HIV positive patients, ${ }^{1-4}$ observing that such patients tend to have advanced AIDS, profoundly depressed CD4 lymphocyte counts, and prior exposure to azole therapy. It is not clear whether these factors are independently associated with the development of fluconazole resistant candidosis in AIDS, as patients with more advanced immunosuppression are likely to have been exposed to more therapy than patients with higher CD4 counts. If increased prior azole exposure were shown to be an independent risk factor for the emergence of resistant candidosis, the prescription of continuous azole prophylaxis might be considered inadvisable.

The objective of this case control study was to evaluate the role of prior azole exposure in the development of resistant candidosis. Control patients were selected with similar CD4 counts and time since first episode of oral candidosis.

and GU Medicine, Chelsea and

Westminster Health Care Trust, London SW10 9NH

J D Cartledge

J Midgley

B G Gazzard

Correspondence to:

Dr Gazzard, Chelsea and

Westminster Hospital, 369

Fulham Road, London

SW10 9NH.

Accepted for publication 27 June 1997

\section{Methods}

\section{PATIENT CHARACTERISTICS}

All patients in the study had clinical signs and symptoms of pseudomembranous candidosis at the time of evaluation, and were selected from the HIV outpatient and inpatient departments of Chelsea and Westminster Hospital.

All patients with fluconazole resistance, defined clinically as failure to eradicate signs and symptoms of thrush following at least 7 days therapy with fluconazole at doses of $100 \mathrm{mg} /$ day or more, were referred to our specialist candida clinic. Patients with complete clinical clearance of candidosis following treatment with $100 \mathrm{mg} /$ day fluconazole for 7 days or less were considered to have fluconazole susceptible thrush.

In vitro resistance or sensitivity to fluconazole was confirmed on samples obtained at the time of clinical evaluation.

The pharmacy department computer, which records all prescriptions from our inpatient and outpatient departments, was checked against the patients' case records to compile a history of azole prescription since first episode of candidosis for each subject. Prescriptions for more than 28 days of azole therapy were considered to be for prophylaxis rather than acute treatment.

Since no azole naive patient attending our unit has been found to have fluconazole resistant candidosis, patients with no prior azole exposure were excluded as potential controls from the study.

Patients with fluconazole susceptible candidosis were matched to cases with resistant thrush for time since first episode of oral candidosis (plus or minus 6 months) and CD4 count at time of fluconazole susceptibility assessment. CD4 counts of the controls were matched to within 10 cells $\times 10^{6} / 1$ if that of the case was $<20$ cells $\times 10^{6} / 1$, within 30 cells 
Table 1 Comparison of prior antifungal prescription in cases with fluconazole resistant candidosis and controls with susceptible candidosis

\begin{tabular}{|c|c|c|c|}
\hline & Cases & Controls & $p$ Value \\
\hline Total days of priorazole therapy & $336(114-1283)$ & $87(21-483)$ & $<0.00001$ \\
\hline \multicolumn{4}{|l|}{ Proportion of days since first candidosis } \\
\hline $\begin{array}{l}\text { prescribed azole therapy } \\
\text { Months of continuous azole prophylaxis }\end{array}$ & $\begin{array}{l}30 \%(22-90 \%) \\
9(4-41)\end{array}$ & $\begin{array}{l}12 \%(0.03-03 \%) \\
0(0-8)\end{array}$ & $<0.0001$ \\
\hline Fluconazole cumulative dose (mg) & $16500(3500-119000)$ & $2900(0-105600)$ & $<0.0001$ \\
\hline Mean daily fluconazole dose (mg)* & $106(48-289)$ & $92(0-360)$ & 0.46 \\
\hline Itraconazole cumulative dose (mg) & $9600(0-42000)$ & $2100(0-44800)$ & 0.07 \\
\hline Mean daily itraconazole dose (mg) ${ }^{\star}$ & $200(0-400)$ & $200(0-400)$ & 0.40 \\
\hline Ketoconazole cumulative dose (mg) & $22400(0-173600)$ & $8400(0-34400)$ & 0.07 \\
\hline Mean daily ketoconazole dose (mg) & $386(0-467)$ & $309(0-400)$ & 0.68 \\
\hline \multicolumn{4}{|l|}{ Cumulative fluconazole dose until 1 month } \\
\hline $\begin{array}{l}\text { before fluconazole failure (mg) } \\
\text { Cumulative fluconazole dose until } 1 \text { year }\end{array}$ & $11700(200-116900)$ & $2900(0-105600)$ & 0.001 \\
\hline before fluconazole failure (mg) & $9800(0-36400)$ & $1400(0-13800)$ & 0.0001 \\
\hline
\end{tabular}

*The mean daily dose of each azole prescribed for each patient was calculated and the median and range for the two groups shown.

$\times 10^{6} / 1$, if that of the case was $20-150$ cells $\times$ $10^{6} / 1$, and within 50 cells $\times 10^{6} / 1$ if that of the case was $>150$ cells $\times 10^{6} / 1$.

SUSCEPTIBILITY TESTING

In vitro susceptibility testing was performed using a technique devised by Odds, ${ }^{5}$ which has been shown to be both sensitive and specific as an indicator of clinically significant resistance to fluconazole. ${ }^{6}$ Briefly, this microplate technique measures the growth of an isolate in a well of CYG (pancreatic casein digest/yeast extract/glucose) broth containing a single concentration of fluconazole $\left(10^{-5} \mathrm{M}: 3 \mu \mathrm{g} / \mathrm{ml}\right)$ and the growth achieved by the same isolate in control well of CYG broth containing no antifungal. The growth in fluconazole is expressed as a percentage of that in the control well to give a value of relative growth in fluconazole. Isolates achieving relative growth in fluconazole exceeding $88 \%$ are associated with candidosis unresponsive to fluconazole in vivo. ${ }^{6}$

\section{STATISTICAL ANALYSIS}

All parametric variables were compared using Student's $t$ test. Non-parametric variables were compared using the Mann-Whitney U test.

\section{Results}

The study sample consisted of 25 patients with fluconazole resistant candidosis and 25 patients with fluconazole susceptible candidosis. CD4 count at time of susceptibility assessment was matched between the two groups, and CD4 count at time of first candidosis did not differ significantly between the two groups (cases, mean 128 cells $\times 10^{6} / 1$; controls, mean 139 cells $\left.\times 10^{6} / 1\right)(p=0.779)$.

The total number of days of azole therapy prescribed before the date of susceptibility evaluation and the proportion of days since first candidosis upon which antifungals were received were significantly longer for the cases than for the controls (table 1) This difference in the number of days of prior azole therapy was largely due to prescriptions for secondary prophylaxis rather than treatment of acute attacks, the patients with fluconazole resistant candidosis having received significantly longer continuous azole prophylaxis than those with responsive thrush (table 1 ). When all prescriptions for secondary prophylaxis (defined as prescription for more than 28 days' treatment) were deducted from the total duration of azole exposure, the median number of days of acute therapy for both cases and controls was the same (84 days).

The total cumulative dose of fluconazole received since first candidosis was significantly higher for those developing fluconazole resistant candidosis than for the control group, even if the fluconazole prescribed in the month or year before susceptibility assessment was excluded from the analysis. There was no difference in the mean daily dose of fluconazole prescribed to the two groups. Although the median total cumulative doses of itraconazole and ketoconazole were higher for the cases than controls, this trend was not statistically significant. Since the prior azole exposure of cases was counted up until the first date of clinical fluconazole failure, the cumulative doses of the other azoles precede this event and do not represent treatment changes in response to fluconazole resistance. None of the patients, cases or controls, had received topical azole therapy.

Concurrent antibiotic therapies were similar for the two groups. All patients were receiving prophylaxis against Pneumocystis either in the form of co-trimoxazole (10 cases, 14 controls), dapsone (10 cases, seven controls), or inhaled pentamidine (five cases, four controls). At the time of sampling the majority (17 cases, 16 controls) were taking no other antibacterial agents, though nine (three cases and six con-

Table 2 Comparison of characteristics of patients infected with fluconazole resistant Candida albicans alone and those infected with fluconazole resistant non-albicans species, either alone (one case) or mixed with $C$ albicans (seven cases)

\begin{tabular}{llc}
\hline & $\begin{array}{l}\text { Patients with resistant } \\
\text { pure Candida albicans } \\
\text { infection }(n=17)\end{array}$ & $\begin{array}{l}\text { Patients with non- } \\
\text { albicans species } \\
\text { isolated }(n=8)\end{array}$ \\
\hline $\begin{array}{l}\text { Median CD4 lymphocyte count cells } \times 10^{6} / 1 \text { at time of } \\
\text { detection of resistance }\end{array}$ & $9(1-59)$ & $15 \cdot 5(2-84)$ \\
$\begin{array}{l}\text { Median total days of prior azole therapy prescribed } \\
\text { Total cumulative dose of prior fluconazole }(\mathrm{mg})\end{array}$ & $378(200-1149)$ & $280(114-1283)$ \\
$13525(6100-119000)$
\end{tabular}


trols) were co-prescribed treatment for mycobacterial infection and eight (five cases, three controls) were given routine antibacterials.

For those patients who developed fluconazole resistant candidosis, there was a significant positive correlation between CD4 count at time of fluconazole failure and days of prior azole exposure $(r=0.45 ; \mathrm{p}=0.02)$ or total cumulative dose of fluconazole $(r=0 \cdot 7, \mathrm{p}$ $<0.001$ ).

Median fluconazole relative growth (growth in fluconazole containing medium expressed as a percentage of that in medium containing no antifungal) was $97 \%$ (range $85-118 \%$ ) for cases and $41 \%$ (range $2-74 \%$ ) for controls. Of the 25 patients with fluconazole resistant candidosis, 17 were infected with fluconazole resistant strains of Candida albicans alone, one with $C$ glabrata alone, and seven with mixtures of $C$ albicans and non-albicans species ( $C$ krusei in three cases; Saccharomyces cereviciae in two cases, $C$ tropicalis in one case, and $C$ glabrata in one case). Although the patients with nonalbicans species present appeared to have higher median CD4 counts, lower duration of prior azole exposure and lower cumulative doses of fluconazole than those with only $C$ albicans isolated, none of these differences were statistically significant (table 2 ).

\section{Discussion}

Although all case-control studies have the potential for hidden biases, the differences observed in this study were striking and the two groups were well matched. The immune status of the two groups would appear similar since although only matched for CD4 count at time of susceptibility assessment they also had similar CD4 counts at time of initial episode of candidosis.

The most relevant finding was that the total cumulative dose of fluconazole received was considerably higher in the group with fluconazole resistance than in the controls, and that this was entirely due to differences in the prescription of continuous fluconazole prophylaxis at doses of $50-100 \mathrm{mg} /$ day. The higher cumulative doses of fluconazole received by those developing resistance did not appear to be attributable to increasing requirements due to intermediate resistance just before drug failure, since the differences remained significant even if the final month or year of azole therapy before drug failure were excluded from the analysis. The patients with fluconazole resistance were exposed to higher doses of other azoles, but not significantly so. The risk of fluconazole resistance may be specifically linked to exposure to this azole; however, in individual cases such resistance occurred after little exposure to fluconazole and greater prior treatment with the other azoles.

Our findings build upon the findings of Johnson et $a l^{7}{ }^{7}$ who demonstrated that HIV positive patients with lower CD4 counts and histories of continuous azole therapy were more inclined to develop fluconazole resistance than less immunosuppressed individuals receiving short courses of antifungal therapy. By matching controls to our cases by CD4 count and duration of recurrent candidosis, the impact of prior azole exposure is more accurately delineated, and shown to be independently related to the emergence of resistance. The retrospective nature of our study makes it possible that other factors may contribute to the differences between the two groups. Exposure to antibacterials, which might encourage candidosis or to agents such as rifampicin or rifabutin which might lessen the efficacy of azole therapy were compared for the cases and controls and found to be similar. In this retrospective study it was not possible to accurately compare the number of episodes of clinical candidosis experienced by each group. If the cases had suffered more frequent recurrent candidosis or more severe symptoms they might require more treatment, or be more inclined to request continuous therapy. These potential factors require prospective evaluation.

The CD4 counts of the resistant cases correlated positively with prior cumulative fluconazole dose, that is those with lower CD4 counts had received less prior fluconazole before developing resistance than those with higher counts. Thus if increasing prior azole exposure is a risk factor for fluconazole resistance, as this study suggests, it would appear that the dose required to predispose to resistance may be lower for more immunosuppressed individuals.

There is a suggestion that non-albicans species resistant to fluconazole were more likely to occur in individuals with higher CD4 counts after less exposure to fluconazole. These differences were not significant, and risk factors for fluconazole resistance due to $C$ albicans compared with non-albicans species are thus being evaluated in a larger study.

Thus, primary or secondary prophylaxis with fluconazole at doses of $50-100 \mathrm{mg} /$ day should only be initiated after careful consideration as this may encourage the development of resistance. Obviously secondary prophylaxis of serious systemic mycoses, particularly cryptococcosis is mandatory. The fluconazole doses used in such situations are higher (200$400 \mathrm{mg} /$ day $)^{8}$ than the doses given to prevent relapsing candidosis for patients in our study. How a higher dosed prophylactic regimen might affect the emergence of resistant candidosis has not yet been evaluated.

A recent study ${ }^{9}$ has indicated that fluconazole $200 \mathrm{mg} /$ day is effective as primary prophylaxis against cryptococcosis, although there was no survival benefit, and the risk of developing such systemic mycoses is low. Breakthrough candidosis was common in this study, and although data regarding susceptibility of Candida species isolated have not been presented fluconazole resistance is a likely cause, if non-compliance with study medication is excluded.

In conclusion, prophylaxis against recurrent candidosis with low doses of fluconazole would appear ill advised since this is associated with the emergence of resistant candido- 
sis. Higher doses may have an impact upon other systemic mycoses though the local risk of such infections needs to be taken into consideration and the risk of resistance emerging with such regimens remains unevaluated.

We wish to thank the Janssen Research Foundation for their part funding of JM's post.

1 Boken DJ, Swindells S, Rinaldi MG. Fluconazole resistant Candida albicans in HIV infection. In: Proceedings of the
ninth international conference on AIDS, Berlin, 1993. ninth international confer

2 Fox R, Neal KR, Leen CLS, Ellis ME, Mandal BK. Fluconazole resistant Candida in AIDS (letter). $\mathcal{f}$ Infect 1991;22:201-2.

3 Kitchen VS, Savage M, Harris JRW. Candida albicans resistance in AIDS (letter). $\mathcal{F}$ Infect 1991;22:204-5.

4 Smith D, Boag F, Midgley J, Gazzard BG. Fluconazoleresistant Candida in AIDS (letter). F Infect 1991;23: 345-6.
5 Odds FC. Antifungal susceptibility testing of Candida species by relative growth measurement at single concenspecies by relative growth measurement at single concentrations of antifungal

6 Cartledge JD, Midgley J, Gazzard BG. Relative growth measurement of Candida species in a single concentration of fluconazole predicts the clinical response to fluconazole in HIV infected patients with oral candidosis. $\mathcal{F}$ Antimicrob Chemother 1996;37:275-83.

7 Johnson EM, Warnock DW, Luker J, Porter SR, Scully C. Emergence of azole drug resistance in Candida species from HIV-infected patients receiving prolonged fluconazole therapy for oral candidosis. $\mathcal{F}$ Antimicrob Chemother 1995;35:103-14.

8 Nelson MR, Fisher M, Cartledge J, Rogers T, Gazzard BG. The role of azoles in the treatment and prophylaxis of Try 651-4.

9 Powderly WG, Finkelstein DM, Feinberg J, Frame P, He W, van der Herst $\mathrm{C}$, et al. A randomised trial comparing fluconazole with clotrimazole troches for the prevention of fungal infections in patients with advanced human immunodeficiency virus infection. $N$ Engl $\mathcal{F}$ Med 1995; 332:700-5. 